\title{
Radio frequency heating induced edge plasma convection: self-consistent simulations and experiments on ASDEX Upgrade
}

\author{
W. Zhang 1,2,3,a), W. Tierens², J-M. Noterdaeme ${ }^{1,2}$, V. Bobkov², D. Aguiam ${ }^{4}$, D. Coster ${ }^{2}$, \\ H. Fuenfgelder ${ }^{2}$, J. Jacquot ${ }^{2}$, R. Ochoukov ${ }^{2}$, A. Silva ${ }^{4}$, L. Colas ${ }^{5}$, A. Křivská ${ }^{6}$, the ASDEX Upgrade \\ team ${ }^{2}$ and the MST1 team*
}

\author{
${ }^{1}$ Applied Physics Department, University of Ghent, Ghent, Belgium \\ ${ }^{2}$ Max-Planck-Institut für Plasmaphysik Garching, Germany \\ ${ }^{3}$ Institute of Plasma Physics, Chinese Academy of Sciences, Hefei, P. R. China \\ ${ }^{4}$ Instituto de Plasmas e Fusão Nuclear, IST, Universidade de Lisboa, Lisboa, Portugal \\ ${ }^{5}$ CEA, IRFM, F-13108 Saint-Paul-Lez-Durance, France \\ ${ }^{6}$ Laboratory for Plasma Physics, ERM/KMS, Brussels, Belgium \\ * see http://www.euro-fusionscipub.org/mst1 \\ ${ }^{a)}$ Corresponding author: wei.zhang @ipp.mpg.de
}

\begin{abstract}
Plasma heating with waves in the Ion Cyclotron Range of Frequency (ICRF) affects the edge plasma and the edge plasma affects the ICRF heating. In simulations, these nonlinear ICRF - edge plasma interactions have been self-consistently simulated by running the EMC3-EIRENE, RAPLICASOL and SSWICH codes in an iterative way on ASDEX Upgrade for the first time. In experiments, the edge plasma convection induced by powered 3-strap antennas is measured with the antenna embedded reflectometers for the first time. Both the simulation and experimental results indicate that the ICRF induced convective cells are most significant on the top and bottom of the antennas; the edge plasma convection induced by 3-strap antennas in optimized antenna feeding configuration (dipole phasing, power ratio between the center and outer straps $\sim 1.5$ ) is smallest among the studied cases. The simulation results also suggest that compared to the 2-strap antennas, the 3-strap antennas can significantly reduce the plasma convection associated with the radio-frequency sheaths, even with unfavorable power balance between the straps in dipole phasing.
\end{abstract}

\section{Introduction}

Plasma heating with Radio Frequency (RF) waves in the lon Cyclotron Range of Frequency (ICRF) is one of the standard heating methods in magnetic controlled fusion devices. It transfers its energy from the antennas to the plasma by coupling to the fast wave. However, the parallel component of electric field $\left(\mathrm{E}_{\|}\right)$in the Scrape-Off Layer (SOL), associated with the excitation of the slow wave, can significantly and nonlinearly enhance the edge plasma potential through sheath rectification: the much lighter electrons are lost much faster to the wall than the ions due to the acceleration of $E_{\|}$; in order to preserve ambipolarity, the plasma develops its potential in a level much larger than the potential across a Debye sheath $\left(\Phi_{B} \approx 3 T_{e}\right)$. A series of effects are then induced due to the enhancement of sheath: in the direction parallel to the magnetic field lines, the large potential drop in the sheath can accelerate ions to be energetic enough to cause physical sputtering; in the direction perpendicular to the magnetic field lines, the potential differences in nearby flux tubes drive ExB drifts (RF convection) in the SOL and 
modify the local density there. The large particle fluxes driven locally to the wall due to the RF convection can increase the intensity of physical sputtering and power dissipation in the sheath.

The many sheath effects - impurity sputtering, RF convection and power dissipation - can be suppressed if the $\mathrm{RF}$ sheath is reduced. To reduce the RF sheath driven close to the 2-strap ICRF antennas, the parallel electric near-field has to be decreased. This can be achieved by minimization of undesired parasitic currents induced in the antenna box by the strap currents. New antennas with a novel approach to reduce those undesired currents through proper phase and amplitude of the current in 3-straps have been installed and validated on ASDEX Upgrade. The recent experimental results have shown that compared to 2-strap antennas, the use of 3-strap antennas reduces the ICRF-specific tungsten release significantly [1]. The experimental observations are also consistent with calculations of the near fields and the rectified potentials [2]. With reflectometers embedded in one 3-strap antenna at different poloidal locations, the density profiles in front of the antenna can be measured when the antenna is either active or passive. The ICRF induced edge plasma convection in different antenna feeding configurations (phasing and power ratio between straps) can thus be studied and compared with the simulation results.

The ICRF waves influence the edge plasma through RF convection, however the edge plasma can also influences ICRF waves and sheath rectification. The physical processes of these nonlinear ICRF - edge plasma interactions are illustrated in figure 1. At first, the parallel electric field $\left(E_{\mid I}\right)$ of the ICRF waves can be known given a plasma density $\left(n_{e}\right)$ and a radiated antenna power. Then with these $n_{e}$ and $E_{||}$, the sheath potential $V_{D C}$ in the vicinity of the antennas is nonlinearly increased through $\mathrm{RF}$ sheath rectification. Subsequently $\mathrm{E} \times \mathrm{B}$ drifts are driven in the SOL due to the spatial inhomogeneity of $V_{D C}$, and the SOL density is modified. The modified SOL density will however further influence $E_{||}$and $V_{D C}$. These physical processes continue on and on until the plasma reaches its final steady state.

The ICRF - edge plasma interactions have long been an interesting topic and extensive studies were carried out $[3,4]$. Large efforts have been made to understand and characterize the RF sheath rectification [5-8] and the RF convection [9-14]. In the previous experiments, the RF convection was measured either by remote diagnostics such as the RFA probes magnetically connected to the powered antennas $[15,16]$ or by density measurements such as the reflectometers [13] and Langmuir probes [11] mounted on the antenna limiters. In our studies, the RF convection in front of the powered antenna is directly measured by the antenna embedded reflectometers. In the previous simulations, the RF convection is mostly investigated non-self-consistently in $2 \mathrm{D}$ geometries. The recent development is the import of the EXB drifts calculated from the measured potential into the EMC3-EIRENE simulations to reproduce the experimental RF convection [14]. In this work, a more comprehensive and advanced simulation method has been developed: the ICRF - edge plasma interactions are studied by running the 3D edge plasma fluid and neutral code EMC3-EIRENE [17], the ICRF wave code RAPLICASOL [18] and the self-consistent sheath code SSWICH [19] in an iterative and self-consistent way. Note that in our simulations, the ICRF induced ExB drifts are the only drifts considered. The drifts due to the ponderomotive potential [20] are in a much smaller level (ponderomotive potential only $\sim 8 \mathrm{~V}$ with $\mathrm{f}_{\mathrm{ICRF}}=35 \mathrm{MHz}$ ) and are not considered in our simulations.

The rest of the paper is organized as follows. In section 2, RF convection is studied with the measurements of the antenna reflectometers. Density modifications induced by powered 3-strap antenna in different antenna feeding configurations are compared. In section 3 , the working flow of the self-consistent simulation loop and the simulation setups are explained. In section 4, the simulation results including $E_{\|}, V_{D C}$ and $n_{e}$ in the final steady-state (when the simulation loop reaches convergence) are discussed. RF convection induced by powered 2-strap and 3-strap antennas are compared. Finally conclusions are made in section 5. 


\section{Experimental results}

In the 2015/2016 experimental campaigns, two tungsten-coated 3-strap antennas (Ant. 2 and Ant. 4) were installed in AUG [1] while the other two antennas (Ant. 1 and Ant. 3) are the old boron-coated 2-strap antennas. In addition, a new multichannel X-mode edge density profile reflectometer system has been installed in Ant. 4 [21]. The current working channels of the reflectometer system are 1, 4 and 8, i.e. the so-called top, middle and bottom antenna reflectometers, respectively (figure 2). The RF convection in front of Ant. 4 can be directly studied from the measurements of these antenna embedded reflectometers.

To investigate the RF convection systematically, a series of L-mode discharges with different antenna feeding configurations have been carried out in AUG. Antennas 2 and 4 or antennas 1 and 3 are powered in pair to provide the whole ICRF power. The ICRF heating scheme is hydrogen minority in deuterium with $\mathrm{f}_{\text {ICRF }}=36.5 \mathrm{MHz}$. The time periods of plasma heating with purely ohmic power are defined as the $\mathrm{OH}$ phases and those with ICRF power (Antennas 2 and 4) are defined as the RF phases. For instance in figure 3, the time periods of $t=[2.4,2.5 \mathrm{~s}$ ], $[2.9,3.0 \mathrm{~s}],[3.4,3.5 \mathrm{~s}]$ are the $\mathrm{OH}$ phases and the time periods of $\mathrm{t}=[2.50,2.65 \mathrm{~s}],[3.00,3.15 \mathrm{~s}],[3.50,3.65 \mathrm{~s}]$ are the RF phases. During the $\mathrm{OH}$ and RF phases, plasma parameters such as the plasma current $\mathrm{I}_{\mathrm{p}}$, safety factor $\mathrm{q}_{95}$, toroidal magnetic field $B_{\mathrm{t}}$, plasma stored energy $\mathrm{W}_{\mathrm{mhd}}$, beta (plasma pressure/magnetic pressure) and plasma outer radius $\mathrm{R}_{\text {outer }}$ are kept almost the same. For instance, during the $\mathrm{OH}$ phase [2.47, 2.49s], these parameters are $\mathrm{I}_{\mathrm{p}}=1.0 \mathrm{MA}, \mathrm{q}_{95}=-2.99, B_{\mathrm{t}}=-1.92 \mathrm{~T}, \mathrm{~W}_{\mathrm{mhd}}=0.10 \mathrm{MW}$, beta=0.16 and $\mathrm{R}_{\text {outer }}=2.16 \mathrm{~m}$; During the adjacent $\mathrm{RF}$ phase $[2.51,2.53 \mathrm{~s}]$, these parameters are $\mathrm{I}_{\mathrm{p}}=1.0 \mathrm{MA}, \mathrm{q}_{95}=-2.95, B_{\mathrm{t}}=-1.92 \mathrm{~T}, \mathrm{~W}_{\mathrm{mhd}}=0.11 \mathrm{MW}$, beta=0.16 and $\mathrm{R}_{\text {outer }}=2.16 \mathrm{~m}$. In particular, the equilibria and the global densities of these two phases are almost the same, as shown in figure 4 . The global densities are measured with the Li-beam diagnostic, which is $\sim 2.1 \mathrm{~m}$ toroidally away from antenna 4 .

Because the global plasma parameters during the $\mathrm{OH}$ and RF phases are almost same, it can be inferred that the local density changes in front of the powered antennas measured with the antenna reflectometers during the $\mathrm{OH}$ and RF phases are due to the RF convection. Thus, the RF convection can be characterized by simply comparing the local density profiles in front of the powered antennas during the $\mathrm{OH}$ and RF phases.

The time traces of a typical experimental discharge (\#33520) are shown in figure 3 . In this discharge, a dipole phasing and a power ratio of $P_{\text {center }} / P_{\text {outer }}=0.1$ (power ratio between the center and the outer two straps) have been set for the 3-strap antennas. It is found that in a certain current step when the plasma current is constant, the SOL density in particular at the top (ref. 1) and bottom (ref. 8) of the antenna 4 during the RF phases are obviously lower than the ones during the $\mathrm{OH}$ phases. The same phenomena are found in other discharges which use other non-optimized antenna feeding configurations. It is worth mentioning that the quick and small density oscillations during the $\mathrm{OH}$ or RF phase is due to the effects of turbulence. The results suggest that the large ICRF induced convective cells are developed in the top and bottom of the antenna when the 3-strap antenna is operated with non-optimized feeding configurations.

To have a more quantitative understanding of the RF convection, the local densities measured with the antenna reflectometers are averaged over the time periods of the $\mathrm{OH}$ and RF phases: the averaged time period for the $\mathrm{OH}$ phase is $[2.47,2.49 \mathrm{~s}]$ and the one for the RF phase is [2.51, 2.53s]. Two discharges are studied, \#33520 is

with a non-optimized power ratio $\left(P_{\text {center }} / P_{\text {outer }}=0.1\right)$ while \#33518 is with an optimized antenna power ratio $\left(P_{\text {center }} / P_{\text {outer }}=1.8\right)$. All the other plasma parameters of the two discharges are almost the same, as shown before. The results (figure 5) indicate that the RF induced density convection in \#33518 is much smaller than that in \#33520, especially in the top of the antenna. This implies that with optimized antenna power ratio, the sheath 
rectification in the antenna box become much smaller. By controlling the phasing and power ratio of the 3-strap antennas, the magnitude of the RF convection can be manipulated.

In the experiments, density profiles are only measured at three poloidal locations of the antenna. To have a comprehensive description of the RF convection, self-consistent simulations in realistic 3D geometry are required. These simulations are discussed in the following two sections.

\section{Simulation setups}

\section{1 simulation loop}

The ICRF - edge plasma interactions require the consideration of the following complex physics: the generation and propagation of the ICRF waves, the RF sheath rectification, the plasma and neutral transport in the SOL and so on. By now no single code can simulate all these physics. Thus, we have used the EMC3-EIRENE, RAPLICASOL and SSWICH codes to construct a self-consistently simulation loop.

EMC3-EIRENE is a code package which couples the EMC3 [17] and the EIRENE [22] codes self-consistently. EMC3 solves a set of time-independent fluid equations for mass, parallel momentum and electron and ion energy, EIRENE computes the kinetic Boltzmann equations for the neutrals. The EMC3 equations including the prescribed drifts terms $\left(\mathbf{V}_{\mathbf{d}}\right)$ are shown as follows [14]:

$\nabla \cdot\left(n_{i} V_{i \|} \vec{b}+\mathbf{n}_{\mathbf{i}} \mathbf{V}_{\mathbf{d}} \overrightarrow{\mathbf{b}}_{\perp}-D_{i} \vec{b}_{\perp} \vec{b}_{\perp} \cdot \nabla n_{i}\right)=S_{p}$

$\nabla \cdot\left(m_{i} n_{i} V_{i \|} V_{i \|} \vec{b}+\mathbf{m}_{\mathbf{i}} \mathbf{n}_{\mathbf{i}} \mathbf{V}_{i \|} \mathbf{V}_{\mathbf{d}} \overrightarrow{\mathbf{b}}_{\perp}-\eta_{\|} \vec{b} \vec{b} \cdot \nabla V_{i \|}-D_{i} \vec{b}_{\perp} \vec{b}_{\perp} \cdot \nabla m_{i} n_{i} V_{i \|}\right)=-\vec{b} \cdot \nabla \mathrm{p}+S_{m}$

$\nabla \cdot\left(\frac{5}{2} n_{e} T_{e} V_{i \|} \vec{b}+\frac{\mathbf{5}}{\mathbf{2}} \mathbf{n}_{\mathbf{e}} \mathbf{T}_{\mathbf{e}} \mathbf{V}_{\mathbf{d}} \overrightarrow{\mathbf{b}}_{\perp}-\kappa_{e} \vec{b} \vec{b} \cdot \nabla T_{e}-\frac{5}{2} T_{e} D_{i} \vec{b}_{\perp} \vec{b}_{\perp} \cdot \nabla n_{e}-\chi_{e} n_{e} \vec{b}_{\perp} \vec{b}_{\perp} \cdot \nabla T_{e}\right)=-k\left(T_{e}-T_{i}\right)+S_{e e}$

$\nabla \cdot\left(\frac{5}{2} n_{i} T_{i} V_{i \|} \vec{b}+\frac{\mathbf{5}}{\mathbf{2}} \mathbf{n}_{\mathbf{i}} \mathbf{T}_{\mathbf{i}} \mathbf{V}_{\mathbf{d}} \overrightarrow{\mathbf{b}}_{\perp}-\kappa_{i} \vec{b} \vec{b} \cdot \nabla T_{i}-\frac{5}{2} T_{i} D_{i} \vec{b}_{\perp} \vec{b}_{\perp} \cdot \nabla n_{i}-\chi_{i} n_{i} \vec{b}_{\perp} \vec{b}_{\perp} \cdot \nabla T_{i}\right)=+k\left(T_{e}-T_{i}\right)+S_{e i}$

In our simulations, the ExB drifts are the only drifts considered and they are calculated with $V_{d}=\vec{E} \times \vec{B} / B^{2}$, where $\vec{E}=-\nabla V_{D C}$. In the first simulation loop, the plasma parameters (such as $n_{e}$ and $T_{e}$ ) calculated with the EMC3-EIRENE code are verified with the experimental data.

RAPLICASOL [18] (Radiofrequency wAve couPLing for lon Cyclotron Antenna in Scrape-Off-Layer) is a finite-element solver based on COMSOL. It solves 3D full-wave Maxwell's equations in frequency-domain in the cold plasma approximation in the neighborhood of realistic antenna geometry. The simulation region is terminated by an absorbing boundary condition at the core plasma side.

Like RAPLICASOL, SSWICH [19, 23] (Self-consistent Sheaths and Waves for lon Cyclotron Heating) is a finite-element solver based on COMSOL. SSWICH self-consistently models both slow wave propagation and nonlinear sheath boundary conditions (SBC) on the antenna limiters. The presently used version of SSWICH-SW makes the assumption that $\mathrm{E}_{\mid \mathrm{I}}$ is only due to the slow wave. 
The workflow of the self-consistent simulation loop is illustrated in figure 6. The iteration loop starts with the EMC3-EIRENE code which generates the 3D edge plasma density for the RAPLICASOL and SSWICH codes. RAPLICASOL then calculates the electromagnetic fields and passes the $E_{\|}$to the SSWICH code. SSWICH is a self-consistent sheath code which computes the sheath rectified $D C$ potential $\left(V_{D C}\right)$ utilizing $E_{\mid l}$ at a surface right in front of the Faraday screen and the 3D edge plasma density. The ExB drifts can be then be calculated from $V_{D C}$. Subsequently the SOL density modifications can be obtained from the EMC3-EIRENE simulations. The new plasma density is then passed to the RAPLICASOL and SSWICH codes for the next iteration. This simulation loop continues until a convergence is reached. In the paper, this convergence is defined as when the changes of plasma density of the adjacent loops are lower than $5 \%$.

\subsection{Computation grid}

In our studies, to best utilize computational resources to solve physics of interest, we have built an EMC3-EIRENE computation grid with a high resolution specified in the low field side of SOL, as shown in figure 7. The computation grid has a toroidal extension of $180^{\circ}$ considering the plasma facing components and the ICRF antennas are $180^{\circ}$ axisymmetric in the toroidal direction. It represents the half of torus from sector one to sector eight (or from sector nine to sector sixteen). Periodic boundary conditions are used to describe the other half of the torus. The use of a toroidal $360^{\circ}$ grid will however increase the computational memory to 200GB which exceeds the maximum amount we can access. To make the EMC3-EIRENE simulations as realistic as possible, plasma facing components such as the divertor and limiters are included in the simulation model.

The RAPLICASOL code uses a tetrahedral grid (a typical grid has about 3 million tetrahedra). It is constructed using COMSOL's mesh construction tools, taking care to select appropriate parameters in different regions of the simulation (for example, the faraday screen is typically meshed somewhat more finely than other regions). The SSWICH code, on the other hand, uses a triangular grid based on the same antenna models.

\subsection{Coordinate transformation}

Both in the SSWICH and RAPLICASOL codes, the flat antenna models (i.e. no toroidal and poloidal curvatures on the surface of the ICRF antennas) have been used. However in the EMC3-EIRENE code, all wall structures are in realistic geometries. Thus, coordinate transformations are necessary when using $V_{D C}$ from SSWICH in the EMC3-EIRENE calculations. The principle of coordinate transformation is illustrated in figure 8 . In the vertical direction, $\mathrm{V}_{\mathrm{DC}}$ is divided into a lot of horizontal slices. These slices are then transformed to the real geometry along the leading edge of the limiter starting from the vertical center of the limiter $(Z=0.0 \mathrm{~m})$ to its two ends. During the transformations, the poloidal distances from the slices to the vertical center $(Z=0.0 \mathrm{~m})$ should not be changed. Meanwhile, the slices are rotated accordingly so that they are always perpendicular to the leading edge of the limiter. For instance, slice $B(Z=0.0 \mathrm{~m})$ is located in the center of the limiter and it is not unchanged during the coordinate transformation. However, slides $A$ and $C$ are transformed in a way such that they are rotated in small angles in order to be still perpendicular to the leading edge of the limiter. Moreover, their distances to slice B (i.e. $\mathrm{d} 1$ and $\mathrm{d} 2$, respectively) are kept unchanged during the coordinate transformation.

\section{Simulation results}

In the self-consistent simulations, the following cases have been investigated: (a) 2-strap antenna with dipole phasing and $\mathrm{P}_{\text {left }} / \mathrm{P}_{\text {right }}=1: 1\left(\mathrm{P}_{\text {left }} / \mathrm{P}_{\text {right }}\right.$ : power ratio between the left and right straps); (b) 3-strap antenna with 
dipole phasing and $P_{\text {center }} / P_{\text {outer }}=3: 2\left(P_{\text {center }} / P_{\text {outer }}\right.$ : power ratio between the center and outer straps); (c) 3-strap antenna with dipole phasing and $P_{\text {center }} / P_{\text {outer }}=1: 9$. A same antenna power of $1 \mathrm{MW}$ is set in these cases. For 3-strap antennas, $P_{\text {center }} / P_{\text {outer }}=3: 2$ is an optimized power ratio while $P_{\text {center }} / P_{\text {outer }}=1: 9$ is a non-optimized one [2]. For 2-strap antennas, $\mathrm{P}_{\text {left }} / \mathrm{P}_{\text {right }}=1: 1$ is a typically power ratio.

The first step of the self-consistent simulations is to generate a background plasma density in the EMC3-EIRENE simulations without including any RF convection. In the simulations, the equilibrium is chosen from \#29498 (a typical L-mode discharge). The separatrix density and the total input power are set as the experimental values, namely $\mathrm{n}_{\mathrm{e}_{-} \text {sep }}=2.0 \times 10^{19} \mathrm{~m}^{-3}$ and $\mathrm{P}_{\text {input }}=1.3 \mathrm{MW}$. The total input power is calculated by subtracting the total radiated power from the total heating power since no impurity is considered in the simulations. Then the background plasma needs to be validated with the experiments by matching the upstream and downstream profiles of the SOL to the experimental ones. This is achieved by modifying the perpendicular particle and energy transport coefficients $D_{\perp}$ and $\chi_{\perp}$. The final results are shown in figure 9 (a), where the simulated profiles including the mid-plane density and temperature, the particle and power fluxes to the divertor targets ( $j_{\text {sat }}$ and $P$ ) are in good agreement with the measurements of the Lithium beam, the Thomson scattering, the divertor Langmuir probe and the infrared camera, respectively. The results (figure 9 (b)) demonstrated that the plasma density in the SOL is rather poloidal homogenous when RF convection is not taken into account. This background plasma density is then used in the RAPLICASOL simulations and it will be compared with the perturbed density when RF convection is included.

Based on the plasma density calculated with the EMC3-EIRENE code, the ICRF wave fields in the SOL can be calculated with the RAPLICASOL code. An example of the real parts of the parallel electric fields $\left(\operatorname{Re}\left(\mathrm{E}_{\|}\right)\right)$is shown in figure 10. As discussed in the introduction, the parallel electric fields in the vicinity of antennas play an essential role on rectifying the sheath, and the most important part of $\operatorname{Re}\left(E_{||}\right)$associated with sheath rectification is the part on the antenna limiters. These $E_{||}$fields on the limiters are generated by the inductive currents on the antenna boxes, while the inductive currents are caused by the currents on the radiated straps. The poloidal variations of $\operatorname{Re}\left(E_{||}\right)$on the antenna straps are due to the existence of Faraday screens. The simulation results clearly indicate that large inhomogeneous $E_{||}$field is generated on the antenna limiters in cases a and $c$ while this value is much smaller in case b. This is because for 3-strap antennas with optimized power ratio (case b), the inductive currents caused by the central strap are mostly canceled by the ones caused by the outer straps [2]; for 3-strap antennas with non-optimized power ratio (case c) or 2-strap antennas (case a), the inductive currents caused by the outer straps are not cancelled.

Based on the parallel electric field of the ICRF waves from RAPLICASOL and the 3D edge plasma density from EMC3-EIRENE, the sheath rectified potential $\left(\mathrm{V}_{D C}\right)$ is then calculated with the SSWICH code. In the SSWICH simulations, the sheath rectified DC potential is calculated on the whole 3D antenna by repeatedly running SSWICH on different horizontal slices. The presently used version of SSWICH is restricted to the slow wave in a 2D domain, hence SSWICH-SW. To get the sheath behaviour throughout the whole 3D antenna, the 2D SSWICH code is run several times on consecutive slices in independent simulations to emulate a pseudo-3D scheme by assuming that poloidal derivatives can be neglected. The slices are not purely horizontal, but in a radial-parallel plane. To satisfy the existence of only a slow wave, the magnetic field can either be parallel or perpendicular to walls. Nonlinear sheath boundary conditions on the antenna limiters are enforced on perpendicular walls to the background magnetic field. The simulation results (figure 11) indicate that $V_{D C}$ is largest for 2-strap antenna (case a), moderate for 3-strap antenna with non-optimized power ratio (case c) and smallest for 3-strap antenna with optimized power ratio (case b). The maximum values of $V_{D C}$ in the cases $a, b, c$ are 127.6, 38.2 and 84.2 volts, respectively (potential of Debye sheath $\sim 30$ volt). The simulation results confirm that the 3-strap antenna with optimized feeding configuration is able to suppress the RF sheath rectification and minimize the $V_{D C}$ values. 
In our simulations, $V_{D C}$ is assumed to be constant along open magnetic field lines. The ExB drifts driven by the differential $V_{D C}$ in nearby flux tubes are calculated with $\overrightarrow{v_{d}}=-\left(\nabla V_{D C} \times \vec{B}\right) / B^{2}$. No other drifts are considered in our simulations. An example of such ExB drifts (for case a) on the top of antenna is shown in figure 12 . Based on these drifts, the calculated steady state densities with EMC3-EIRENE are shown in figure 13. The results indicate that in the radial direction, the RF convective cells are formed in a layer about $2 \mathrm{~cm}$ in front of the Faraday Screen; in the poloidal direction, they are mainly developed in the top and bottom of the antennas. Driven in the clockwise direction, the plasma flows are very complex especially when two convective cells are connected to each other. The largest drift exists where the gradient of the potential is largest. The simulation results suggest that the local density convections induced by powered 2-strap antennas (case a) are most significant. They become smaller for powered 3-strap with non-optimized power ratio (case c) and are lowest for powered 3-strap antennas with optimized power ratio (case b). This indicates that the RF convection can indeed be minimized with 3-strap antennas operated in optimized feeding configurations. Local density depletions are found in the center of the convective cells and the corresponding density accumulations are observed in the margin of convective cells. In particular, these density accumulations are often found where the convective cells interact with the wall.

To have a detailed understanding of the density convections, density differences between the perturbed density in figure 13 and the unperturbed density in figure 9 (the background plasma density) have been made. The results are shown in figure 14, where regions of density accumulations are shown in red color while regions of density depletions are shown in blue color. The results confirm that the density convections in case a and case $c$ are much larger than those in case b. The most obvious density convections are developed in the top and bottom of the powered antennas.

The results (figures 10-14) shown previously are all in their final steady state when convergences of the simulations have been reached. An example of convergence of the simulated case a is shown in Figure 15 . When the ExB drifts are applied in the simulations, the SOL density in loop 2 changes significantly compared to the unperturbed density in loop 1 . The SOL density is modified due to the nonlinear ICRF-edge plasma interactions in loop2 and loop3. From loop3 to loop4, the density modifications are already in a significant small level (lower than $5 \%)$. At this point, the simulation loops are considered as converged.

The simulation results are consistent with the experimental ones on the following points: 1) the RF convection in the top and bottom of the powered antennas is often much larger than that in the middle of antennas; 2 ) the RF convection induced by powered 3-trap antennas with optimized antenna feeding configurations is much smaller than the one induced by powered 3-strap antennas with non-optimized antenna feeding configurations; 3) the peak density modification in simulations (figure 14) is of the order of 2.e18, which is the same order as in the experimental result (figure 5). However, the simulations and experiments do have a divergence in terms of the radial extension of the RF convection: the RF convection observed in the experiments is extended to the pedestal region while in the simulations this extension is only about $2 \mathrm{~cm}$ in front of the antenna. From the previous experimental analysis with the RFA probes, the RF convective cells are expected to have a radial extension of $2 \mathrm{~cm}$ in front of the antennas in L-mode plasmas [16]. This is more in line with our simulation results. It is suspected that some mechanisms which play a role in reshaping the density profiles are not considered in our simulations. For instance, the interaction between the blobs and the convective cells can have a large influence on the density profiles [24]. However this mechanism is not included in our simulations. 


\section{Conclusions}

The nonlinear ICRF - edge plasma interactions have been self-consistently simulated for the first time by running the EMC3-EIRENE, RAPLICASOL and SSWICH codes in an iterative way. The RF convections induced by powered 2-strap and 3-strap antennas in different antenna feeding configurations are investigated. In addition, we have used the antenna embedded reflectometers to measure the density modifications in front of the powered antenna.

Both the simulation and experimental results indicate that the RF convection in the top and bottom of the antennas is much more significant compared to the one in the middle of antenna. Different antenna feeding configurations (phasing and power ratio between the straps) result in different sheath potential and different RF convections. The density convection induced by the powered 2-strap antennas with typical antenna feeding configuration (dipole phasing, power ratio between the left and right straps $\sim 1$ ) is found to be largest among the studied cases. Density convections induced by 3-strap antenna with optimized antenna feeding configuration (dipole phasing, power ratio between the center and outer straps $\sim 1.5$ ) is much smaller than that induced by 3-strap antenna with non-optimized antenna feeding configurations.

The measured density profiles with the antenna embedded reflectometers are consistent with the simulation results except the following discrepancies. The first discrepancy is that in experiments we mostly see density depletions while in simulations we both see density depletions and density accumulations. The second discrepancy is that the radial extension of the RF convection is different. In experiments it is found that the RF convection can reach the pedestal region while in simulations it only exists in a layer about $2 \mathrm{~cm}$ in front of the antenna. It is suspected that this is due to some other mechanisms such as the interactions between the blobs and convective cells in the SOL which have not been considered in our simulations. These mechanisms can indeed affect the radial and poloidal plasma flows and modify the SOL density. Further studies with dedicated experiments and simulations on AUG are expected to address these issues.

Acknowledgments: This work has been carried out within the framework of the EUROfusion Consortium and has received funding from the Euroatom research and training programme 2014-2018 under grant agreement No 633053. The views and opinions expressed herein do not necessarily reflect those of the European Commission. 


\section{References}

[1] Bobkov V. et al 2016 Nuclear Fusion 56084001

[2] Bobkov V. et al 2017 Plasma Phys. Control. Fusion 59014022

[3] Noterdaeme J. M. et al 1993 Plasma Physics and Controlled Fusion 35 1481-511

[4] Myra J. R. et al 2006 Nuclear Fusion 46 S455-S68

[5] Butler H. S. et al 1963 Physics of Fluids 6 1346-55

[6] Perkins F. W. 1989 Nuclear Fusion 29 583-92

[7] D'Ippolito D. A. et al 2006 Physics of Plasmas 13102508

[8] Myra J. R. et al 1994 Physics of Plasmas 1 2890-900

[9] D'Ippolito D. A. et al 1993 Physics of Fluids B-Plasma Physics 5 3603-17

[10] D'Ippolito D. A. et al 1998 Nuclear Fusion 38 1543-63

[11] Becoulet M. et al 2002 Physics of Plasmas 9 2619-32

[12] Colas L. et al 2015 Journal of Nuclear Materials 463 735-8

[13] Lau C. et al 2013 Plasma Physics and Controlled Fusion 55095003

[14] Zhang W. et al 2016 Plasma Phys. Control. Fusion 58095005

[15] Kubič M. et al 2013 Journal of Nuclear Materials 438 S509-S12

[16] Colas L. et al 2014 2-Dimensional Mapping of ICRF-Induced Scrape-Off Layer Modifications with a Retarding Field Analyser on ASDEX-Upgrade 20th Topical Conference on Radio Frequency Power in Plasmas (Sorrento, ITALY 2013) 1580 259-62 http://dx.doi.org/10.1063/1.4864537

[17] Feng Y. et al 2004 Contributions to Plasma Physics 44 57-69

[18] Jacquot J. et al 2015 AIP Conference Proceedings 1689050008

[19] Jacquot J. et al 2014 Physics of Plasmas 21061509

[20] Van Eester D. et al 2013 Plasma Physics and Controlled Fusion 55025002

[21] Aguiam D. E. et al 2016 Review of Scientific Instruments $8711 \mathrm{E} 722$

[22] Reiter D. et al 2005 Fusion Science and Technology 47 172-86

[23] Colas L. et al 2012 Physics of Plasmas 19092505

[24] D'Ippolito D. A. et al 2005 AIP Conference Proceedings 787 222-5 


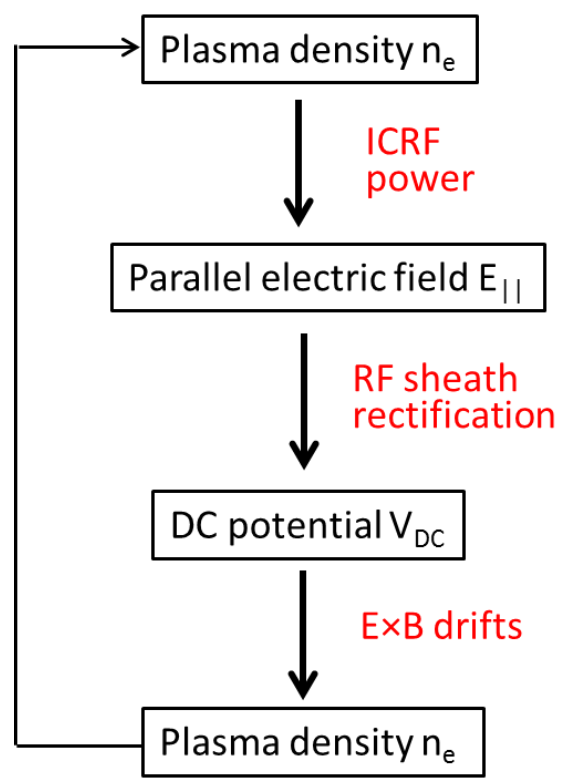

Figure 1. Physical processes of the nonlinear ICRF - edge plasma interactions.

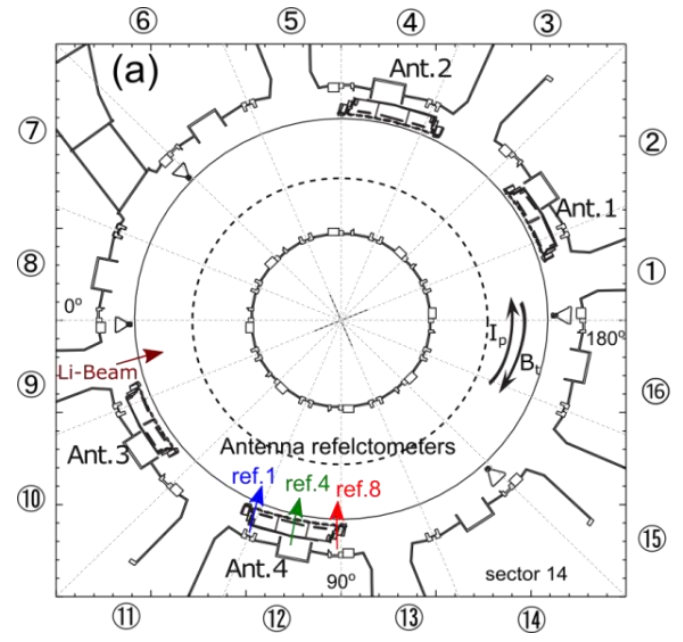

(b) antenna reflectometers

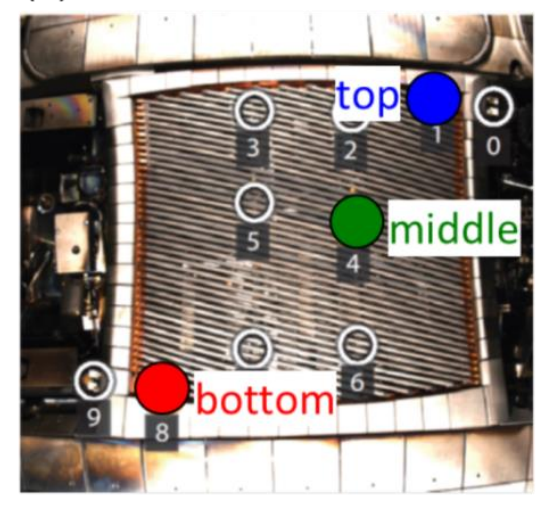

Figure 2. (a) Top view of AUG showing the toroidal positions of the antennas and density diagnostics. (b) Spatial locations of the top (ref.1), middle (ref. 4) and bottom (ref. 8) antenna embedded reflectometers. 


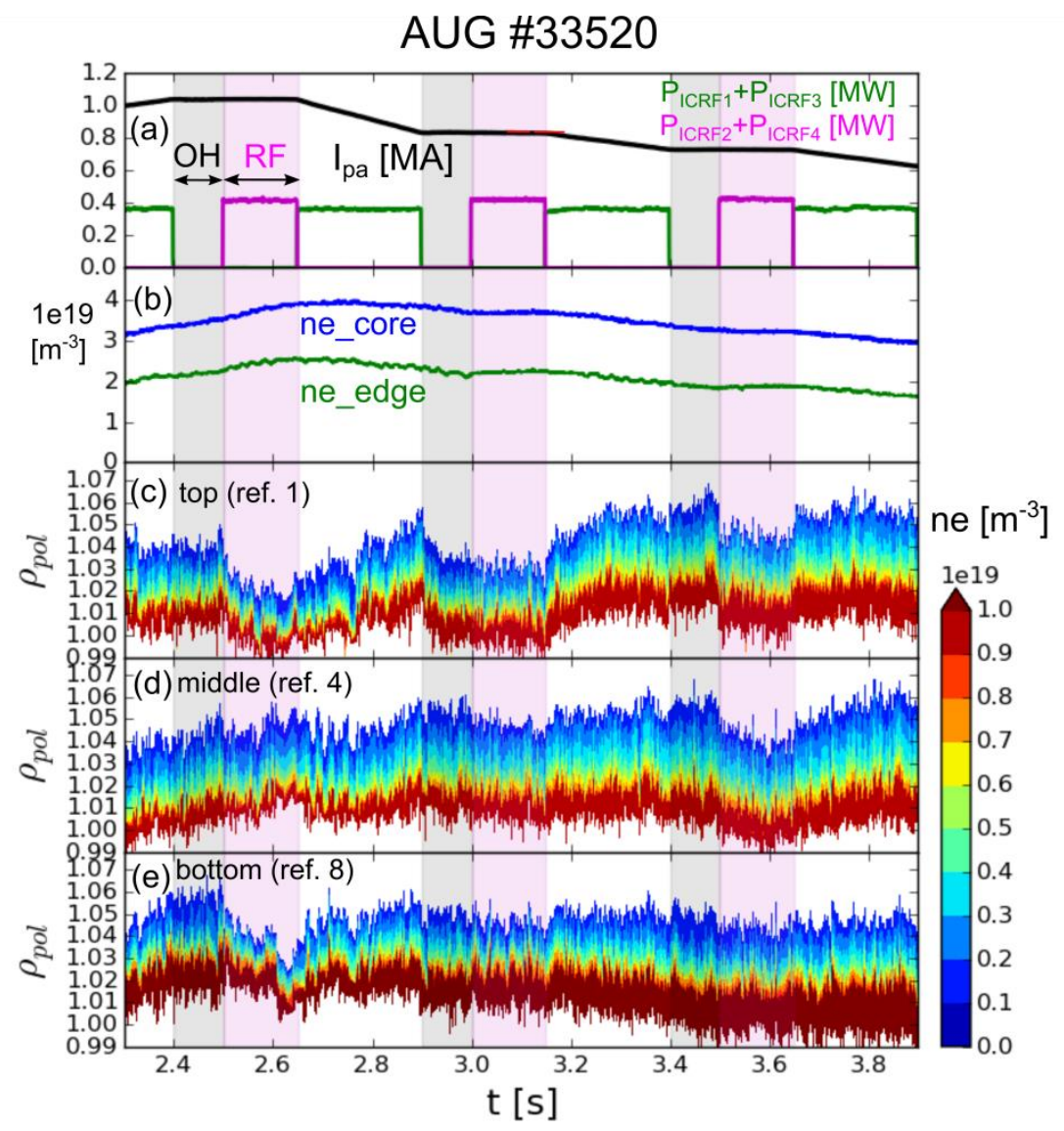

Figure 3. Time traces of plasma parameters including the plasma current, ICRF heating power, core and edge line integrated densities and local density measurements of the antenna embedded reflectometers.

(a) $-2.48 \mathrm{~s}(\mathrm{OH}$ phase)

(b) New Lithium beam
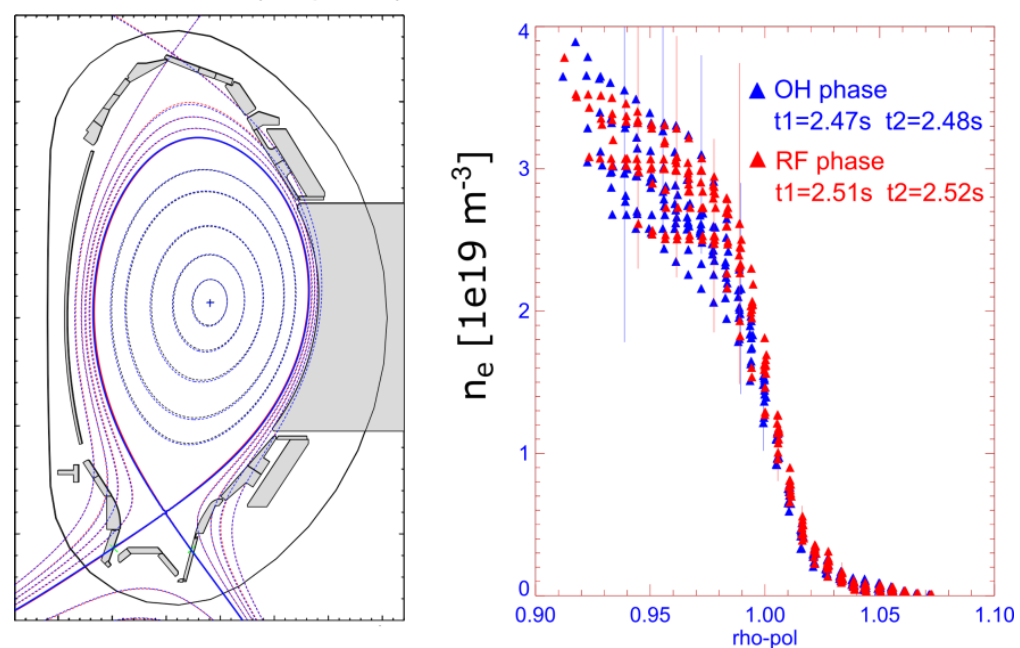

Figure 4. (a) Equilibriums and (b) global density profiles measured with Lithium beam during the RF and $\mathrm{OH}$ phases. The parameters of the $\mathrm{RF}$ and $\mathrm{OH}$ phases are shown in red and blue color, respectively. 

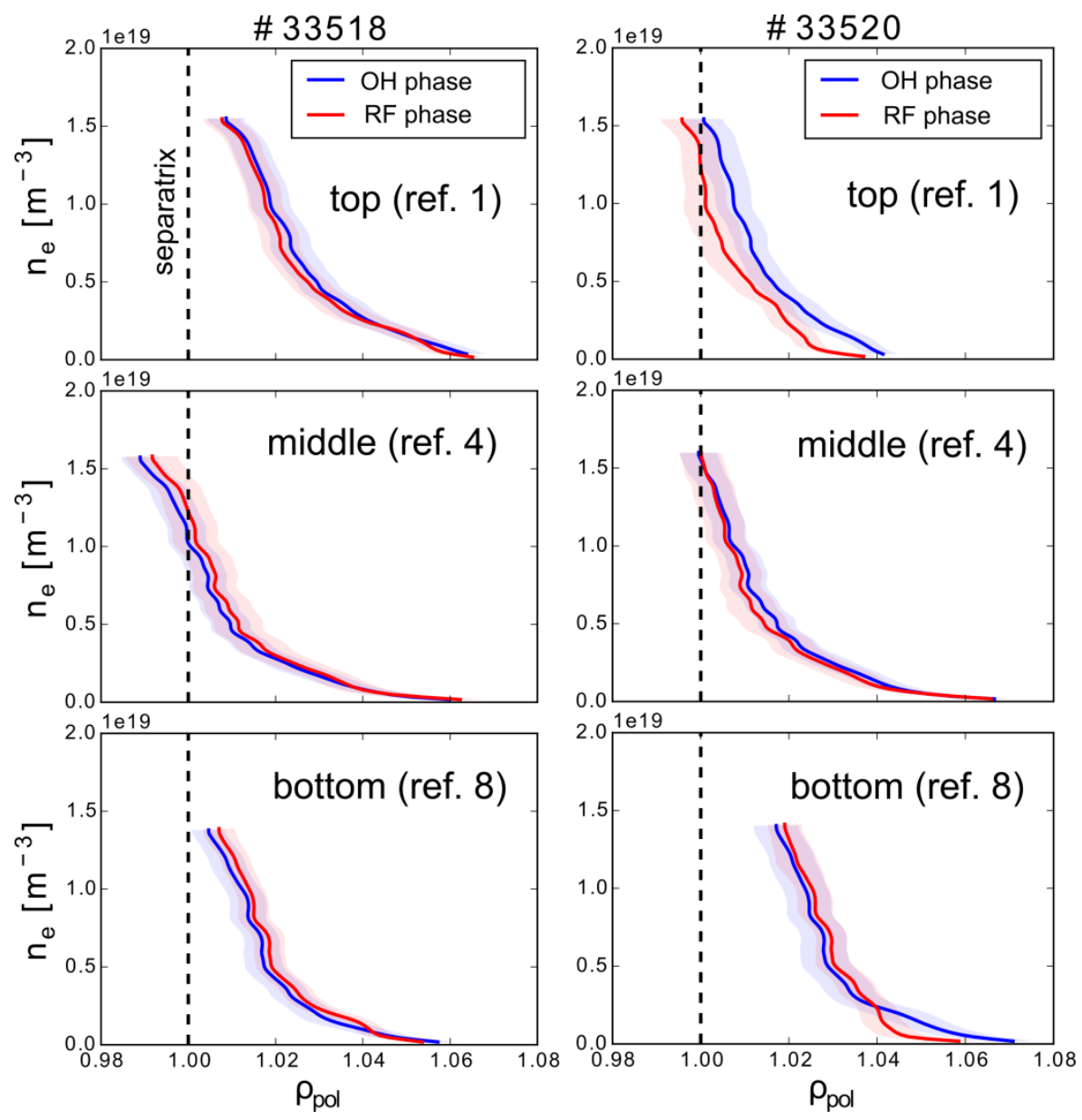

Figure 5. Density profiles of \#33518 (with optimized antenna power ratio) and \#33520 (with non-optimized antenna power ratio) measured with the antenna embedded reflectometers. They are averaged in the time periods of $\mathrm{t}_{\mathrm{OH}}=[2.47,2.49 \mathrm{~s}]$ for the $\mathrm{OH}$ phase and $\mathrm{t}_{\mathrm{RF}}=[2.51,2.53 \mathrm{~s}]$ for the RF phase.

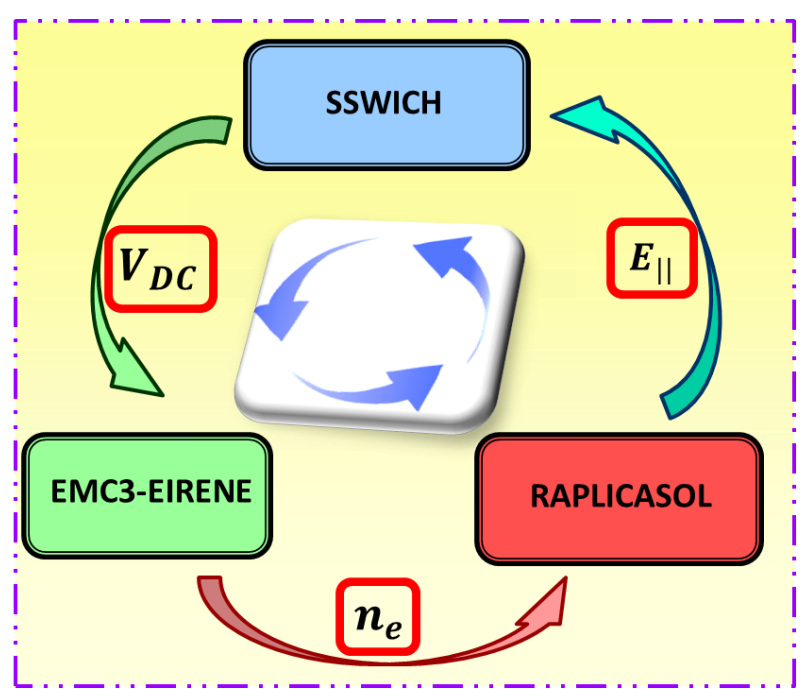

Figure 6. The workflow of the self-consistent simulation loop. 

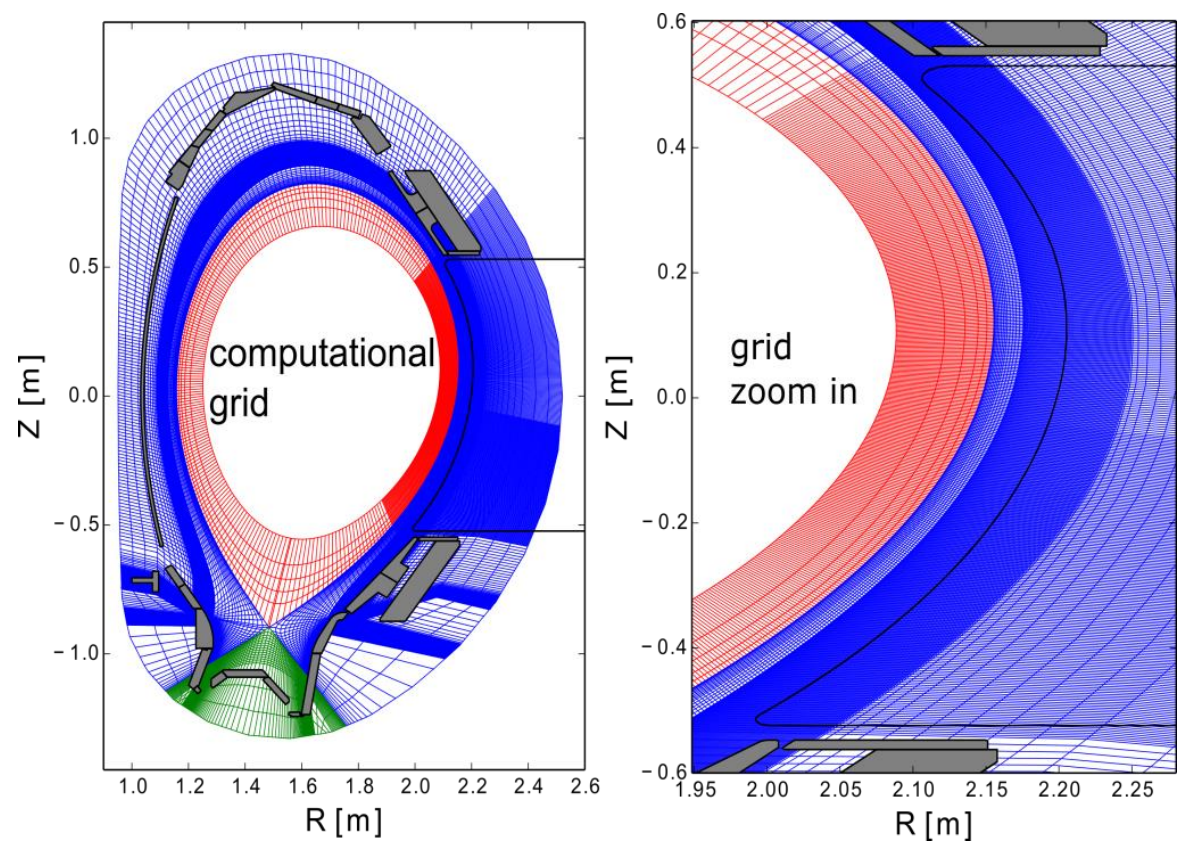

Figure 7. EMC3-EIRENE computation grid. Left: poloidal cross-section of the computation grid at $\Phi=73.83^{\circ}$. Right: zoom in of the computation grid in the low field side.

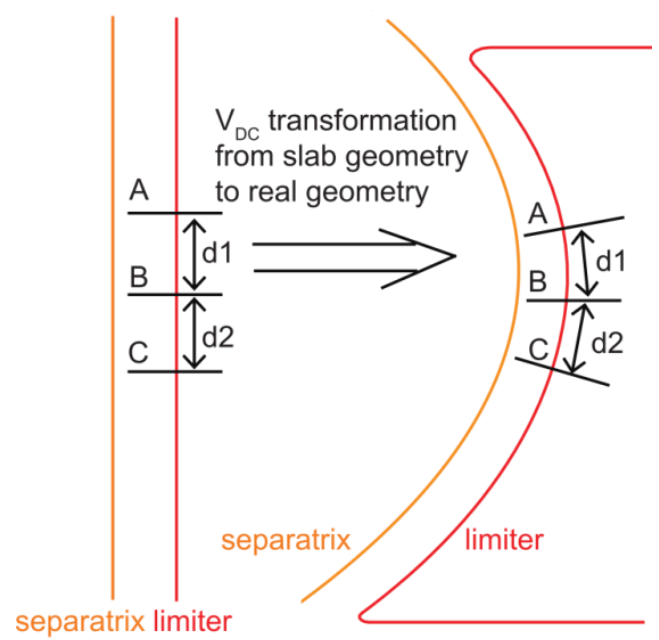

Figure 8. Transformation of $\mathrm{V}_{\mathrm{DC}}$ from slab geometry to realistic geometry. The SSWICH code uses slab antenna geometry while the EMC3-EIRENE uses realistic antenna geometry. 
(a) \#29498@2.95s



(b) Backgroud plasma

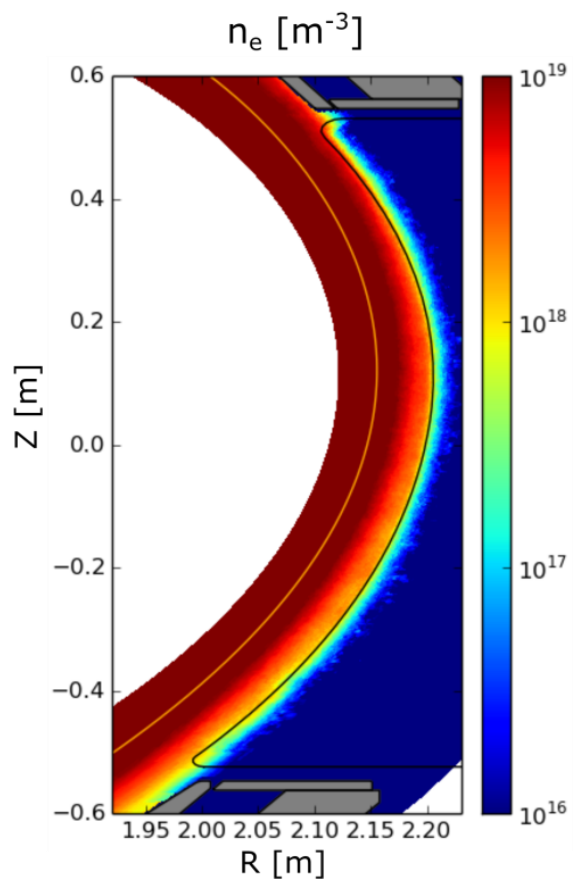

Figure 9. (a) validations of the upstream profiles (mid-plane density and temperature) and downstream profiles $\left(\mathrm{J}_{\text {sat }}\right.$ and $\left.\mathrm{P}\right)$ of the background plasma with experimental measurements. Figure reproduced with permission from [14]. (b) the validated background plasma density.

(a) 2-strap

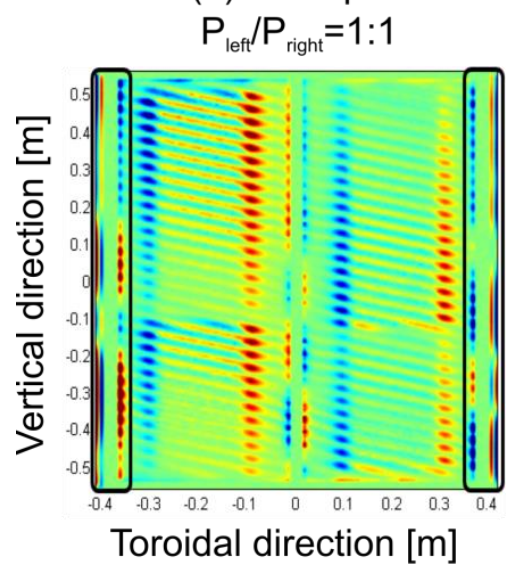

(b) 3-strap

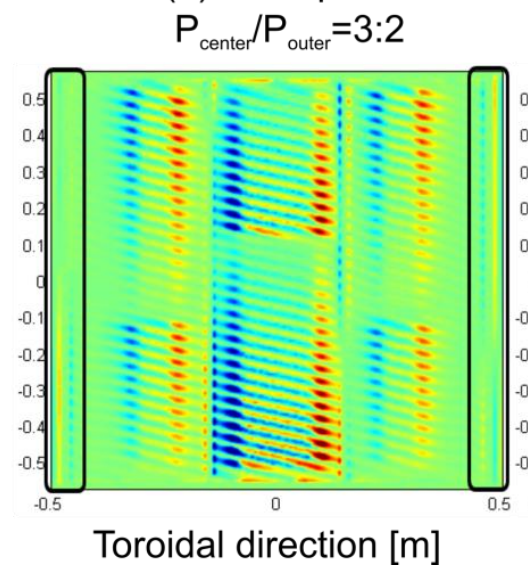

(c) 3-strap

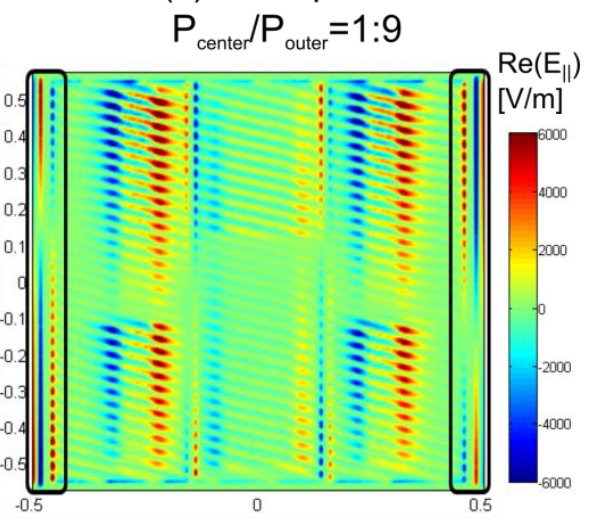

Toroidal direction [m]

Figure 10. Real part of the parallel electric fields calculated with the RAPLICASOL code. The electric field map is on a surface right in front of the Faraday bars. 

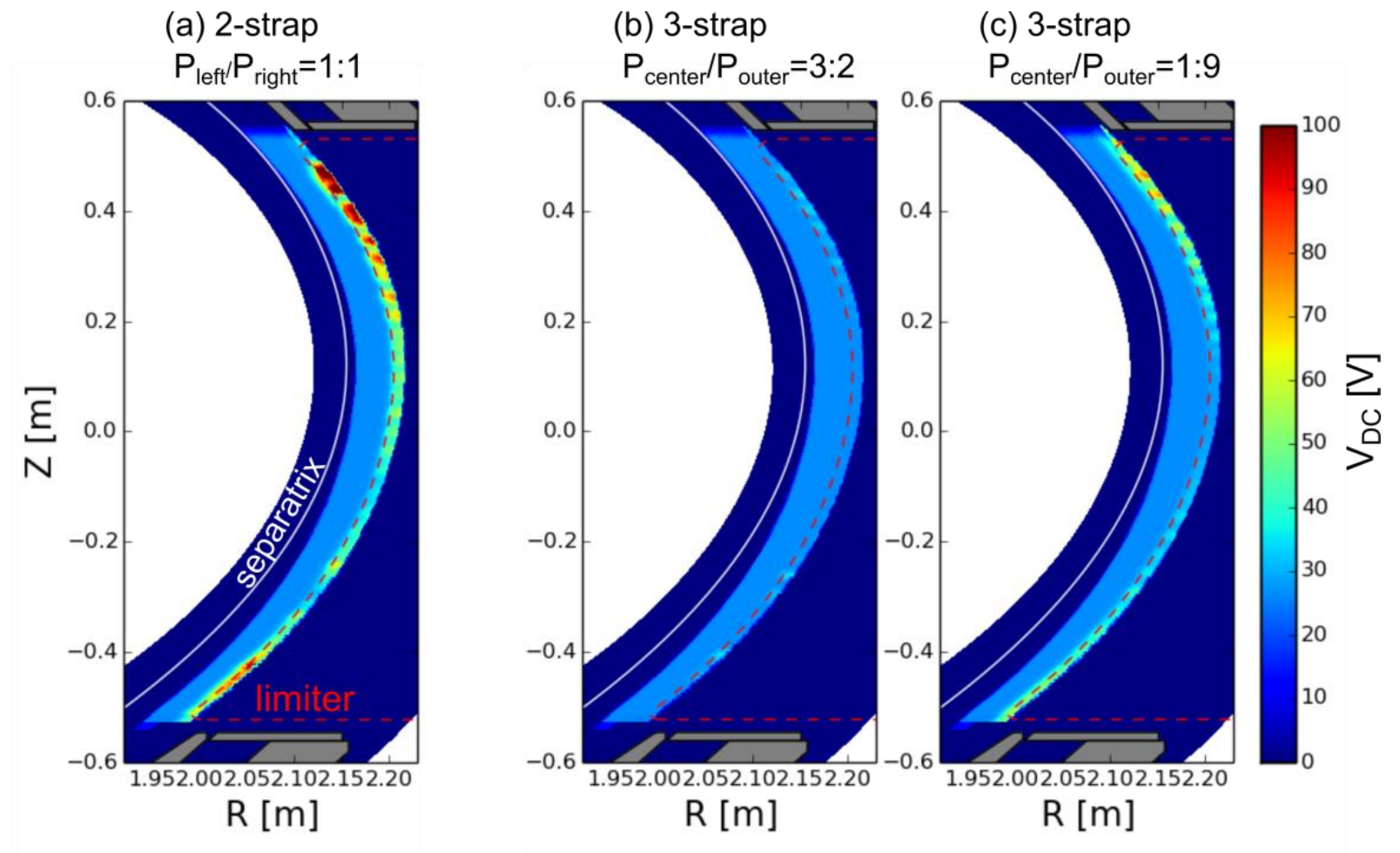

Figure 11. DC potential $\left(V_{D C}\right)$ calculated with the SSWICH code. The white line represents the separatrix and the red dashed line means the antenna limiter. The poloidal cross-sections are chosen at the middle of antenna 4 $\left(\Phi=73.83^{\circ}\right)$. 


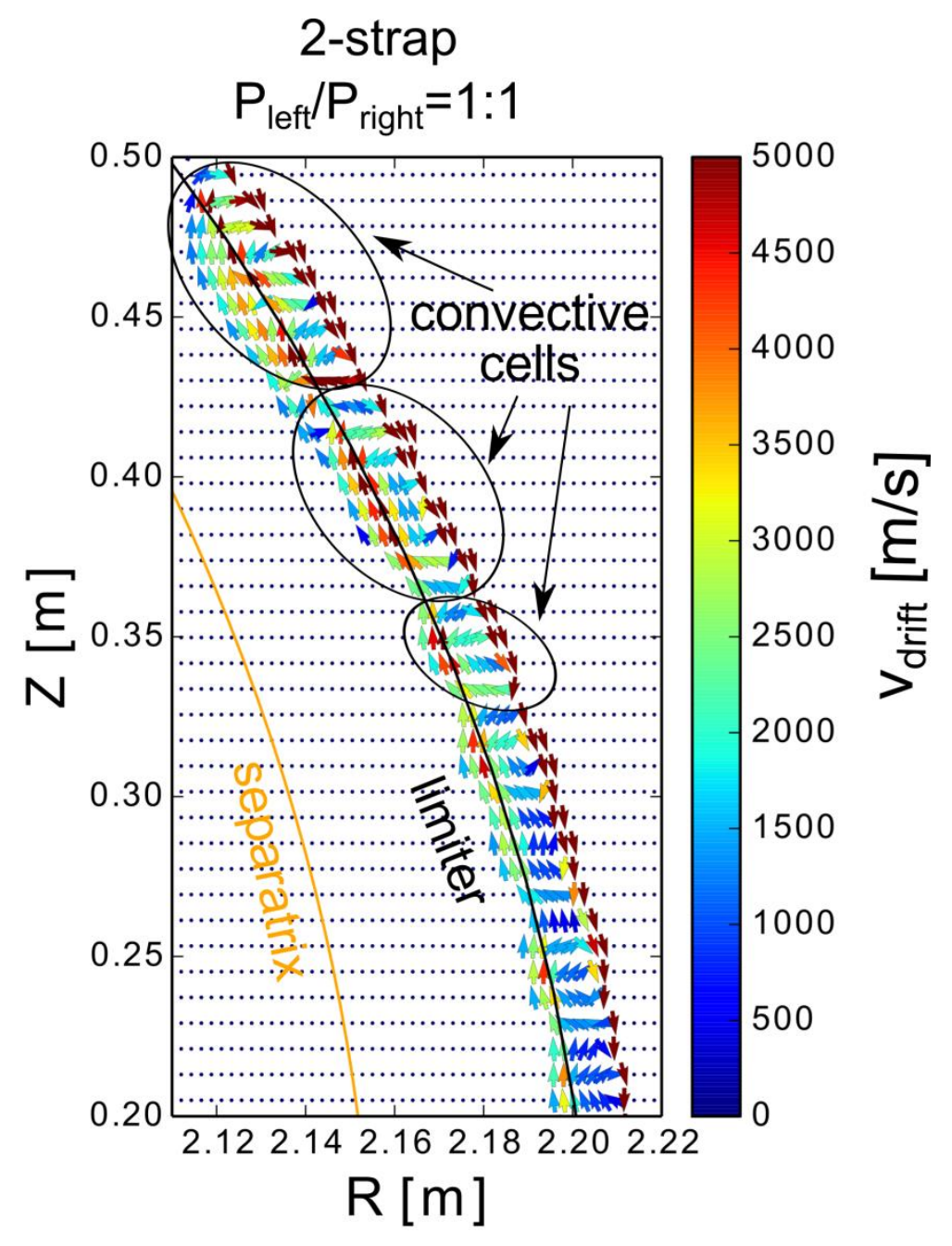

Figure 12. $E \times B$ drifts calculated in the top of antenna with $V_{D C}$ from figure 11(a). 

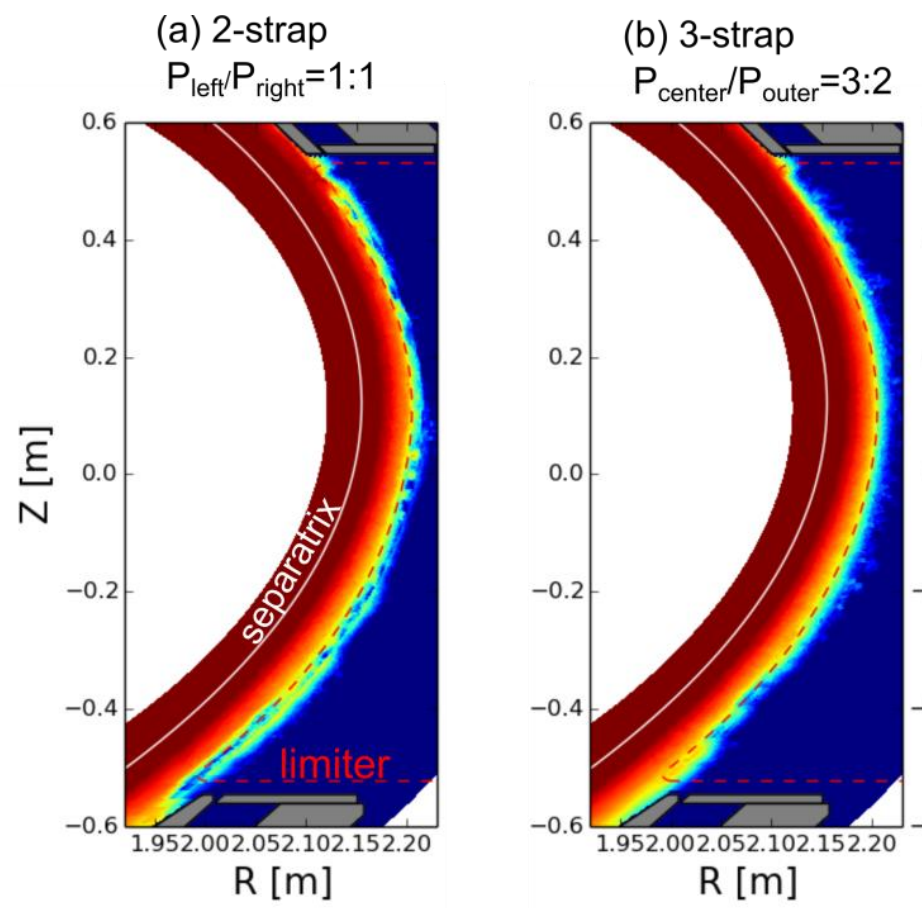

(c) 3-strap

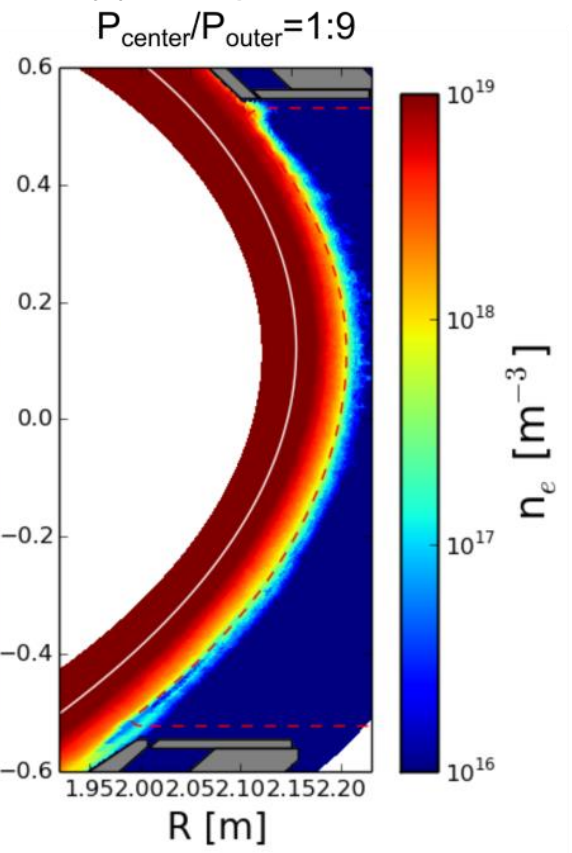

Figure 13. Density redistributions calculated with the EMC3-EIRENE code. Density depletions and accumulations are found in the neighbourhood of the antenna. The poloidal cross-sections are taken in the middle of antenna 4 $\left(\Phi=73.83^{\circ}\right)$.
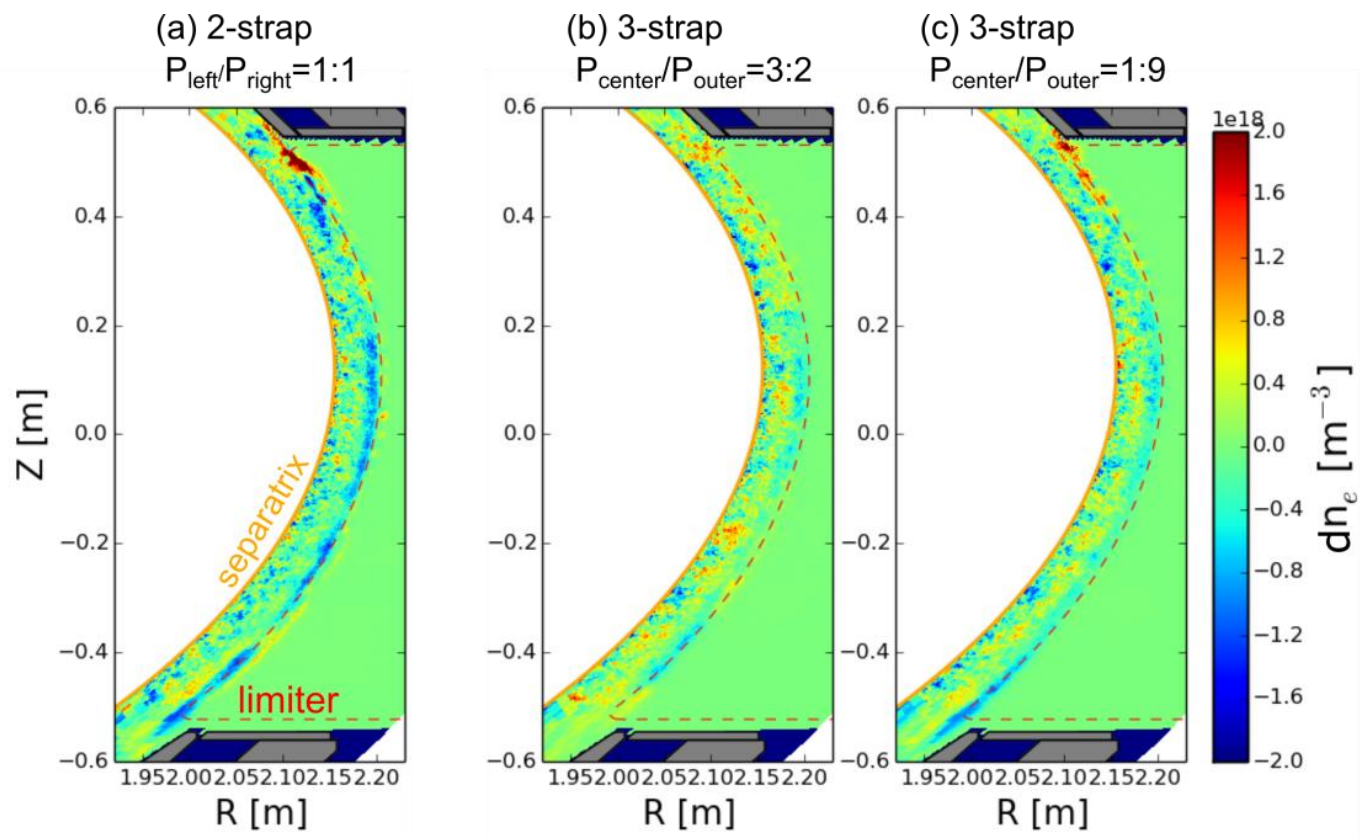

Figure 14. Density differences show regions of density accumulations and density depletions due to RF convection. The poloidal cross-sections are taken at the middle of antenna $4\left(\Phi=73.83^{\circ}\right)$. 




Figure 15. Convergence of the iteration loops for the studied case a (2-stap antenna with dipole phasing and $\left.\mathrm{P}_{\text {left }} / \mathrm{P}_{\text {right }}=1: 1\right)$. 